\title{
ON THE FLUX OF DIFFUSING MATTER THROUGH BOUNDARIES
}

\author{
A. McNABB
}

Introduction. Suppose $C(x, t)$, the concentration of a substance diffusing in a slab of homogeneous material of thickness $a$, is determined by the system

$$
\frac{\partial C}{\partial t}=K \frac{\partial^{2} C}{\partial x^{2}}, \quad 0<x<a
$$

$C=C_{0}$ at $x=0 ; C=0$ at $x=a$ for $t>0 ; C=0$ at $t=0$.

The quantity of matter

$$
Q(t)=\int_{0}^{t}\left[K \frac{\partial C}{\partial x}\right]_{x=a} d t
$$

which has passed through the medium in time $t$ approaches an asymptote of slope $C_{0} K / a$ as $t$ tends to infinity, intercepting the $t$-axis at a point $t^{\prime}=a / 6 K$. This intercept has been used by Daynes [1] and Barrer [2, p. 19] as the basis of a method for obtaining the diffusion constant $K$. In this note we show how to calculate the slope and intercept of the asymptote in the more general case where

$$
Q(t)=\int_{0}^{t}\left\{\iint_{S_{0}} K(u) \operatorname{grad} u \cdot d s\right\} d t
$$

is the quantity of diffusing substance which has passed through the part $S_{0}$ of the boundary $\partial R$ of a region $R$ in time $t$ and $u$ is determined by the system

$$
\operatorname{div}[K(u) \operatorname{grad} u]-\frac{\partial u}{\partial t}=\frac{\partial w}{\partial t}=f(u, w)
$$

in $R$ for $t>0 ; u=u_{0}, w=w_{0}$ in $\bar{R}$, the closure of $R$, at $t=0 ; u=C(x, y, z, t)$ on $\partial R$ for $t>0$. Such systems govern the diffusion of a substance $U$ of concentration $u(x, y, z, t)$ which diffuses through a medium in accord with Fick's first law and simultaneously interacts with an immobile phase $W$. If $w(x, y, z, t)$ is the local concentration of $W$, the principle of conservation of matter requires that $u$ and $w$ satisfy the differential equation

Received by the editors December 27, 1960 and, in revised form, May 19, 1961. 


$$
\frac{\partial u}{\partial t}+\frac{\partial w}{\partial t}=\operatorname{div}[K(u) \operatorname{grad} u]
$$

throughout the region $R$ defined by the medium. The second equation

$$
\frac{\partial w}{\partial t}=f(u, w)
$$

describes the local law governing the rate of interchange of matter between the two phases $U$ and $W$.

Calculation of the asymptote. Let $D$ denote the open region $\{(x, y, z, t):(x, y, z) \in R, t>0\}$ and $\bar{D}$ its closure. Suppose $u$ and $w$ are continuous in $\bar{D}$, while in $D, \partial w / \partial t$ is continuous, the second order space derivatives and the $t$-derivative of $u$ are also continuous and these derivatives satisfy the pair of equations (3) and (4). The function $K(u)$ is assumed to be positive and have a continuous derivative for all admissible values of $u$. In addition, suppose $u_{\infty}$, $w_{\infty}$ are defined and continuous in $\bar{R}$ with continuous derivatives satisfying the system

$$
\operatorname{div}\left[K\left(u_{\infty}\right) \operatorname{grad} u_{\infty}\right]=0=f\left(u_{\infty}, w_{\infty}\right)
$$

in $R$, while $u$ and $w$ and their derivatives approach the corresponding values of $u_{\infty}, w_{\infty}$ and their respective derivatives at each point of $R$.

The flux of $U$ over a part $S_{0}$ of the boundary $\partial R$ is

$$
F(t)=-\iint_{S_{0}}[K(u) \operatorname{grad} u] \cdot d s
$$

and as $t$ tends to infinity this approaches the constant value

$$
F(\infty)=-\iint_{S_{0}}\left[K\left(u_{\infty}\right) \operatorname{grad} u_{\infty}\right] \cdot d s
$$

The amount of phase $U$ which has passed through $S_{0}$ in time $t$ is

$$
Q(t)=\int_{0}^{t} F(\tau) d \tau=F(\infty) t-\int_{0}^{t}[F(\infty)-F(\tau)] d \tau .
$$

It will be shown that $\int_{0}^{t}[F(\infty)-F(\tau)] d \tau$ tends to a constant $A$ as $t$ tends to infinity.

Let us define $\phi(x, y, z, t)$ as $\int_{u(x, y, z, t)}^{u(x, y, z, \infty)} K(\theta) d \theta$ and $\psi(x, y, z, t)$ as $\int_{0}^{t} \phi(x, y, z, \tau) d \tau$ in $\bar{D}$, so that 


$$
\begin{aligned}
\nabla^{2} \psi & =-\int_{0}^{t} \operatorname{div}\left\{K(u) \operatorname{grad} u-K\left(u_{\infty}\right) \operatorname{grad} u_{\infty}\right\} d \tau \\
& =-\int_{0}^{t}\left[\frac{\partial u}{\partial \tau}+\frac{\partial w}{\partial \tau}\right] d \tau \\
& =u_{0}+w_{0}-u(x, y, z, t)-w(x, y, z, t)
\end{aligned}
$$

in $R$ for each value of $t$, while on the boundary $\partial R$,

$$
\begin{aligned}
\psi & =\int_{0}^{t} \int_{u(x, y, z, \tau)}^{u(x, y, z, \infty)} K(\theta) d \theta d \tau \\
& =\left[\tau \int_{u(x, y, z, \tau)}^{u(x, y, z, \infty)} K(\theta) d \theta\right]_{0}^{t}+\int_{0}^{t} \tau K(u) \frac{\partial u}{\partial \tau} d \tau .
\end{aligned}
$$

In the limit as $t$ tends to infinity, $\psi$ tends to a function $\psi_{\infty}$ defined by the system

$$
\begin{aligned}
\nabla^{2} \psi_{\infty} & =u_{0}+w_{0}-u_{\infty}-w_{\infty} \text { in } R \\
\psi_{\infty} & =\int_{0}^{\infty} \tau K(u) \frac{\partial u}{\partial \tau} d \tau \text { on } \partial R
\end{aligned}
$$

provided this last integral exists and is continuous at each point of $\partial R$, and the boundary is sufficiently smooth.

Now

$$
\begin{aligned}
\int_{0}^{t}[F(\infty)-F(\tau)] d \tau \\
=-\iint_{s_{0}}\left\{\int_{0}^{t}\left[K\left(u_{\infty}\right) \operatorname{grad} u_{\infty}-K(u) \operatorname{grad} u\right] d \tau\right\} \cdot d s \\
=-\iint_{s_{0}}\left\{\int_{0}^{t} \operatorname{grad} \phi d \tau\right\} \cdot d s \\
=-\iint_{s_{0}} \operatorname{grad} \psi \cdot d s
\end{aligned}
$$

and as $t$ tends to infinity, the right hand side approaches the value $-\iint_{s_{0}} \operatorname{grad} \psi_{\infty} \cdot d s$.

Summarizing these results, we see that

$$
Q(t) \sim F(\infty) t-A
$$

where 


$$
F(\infty)=-\iint_{s_{0}}\left[K\left(u_{\infty}\right) \operatorname{grad} u_{\infty}\right] \cdot d s
$$

and

$$
A=-\iint_{S_{0}} \operatorname{grad} \psi_{\infty} \cdot d s
$$

The function $u_{\infty}$ is determined by the system

$$
\operatorname{div}\left[K\left(u_{\infty}\right) \operatorname{grad} u_{\infty}\right]=0=f\left(u_{\infty}, w_{\infty}\right) \text { in } R,
$$

where on $\partial R$,

$$
u_{\infty}=\lim _{t \rightarrow \infty} u(x, y, z, t)=\lim _{t \rightarrow \infty} C(x, y, z, t),
$$

while the function $\psi_{\infty}$ satisfies

$$
\nabla^{2} \psi_{\infty}=u_{0}+w_{0}-u_{\infty}-w_{\infty} \text { in } R,
$$

and the boundary conditions

$$
\psi_{\infty}=\int_{0}^{\infty} \int_{u(x, y, z, \tau)}^{u(x, y, z, \infty)} K(\theta) d \theta d \tau=\int_{0}^{\infty} \tau K(u) \frac{\partial u}{\partial \tau} d \tau
$$

on $\partial R$.

In the case where $S_{0}$ coincides with the whole surface $\partial R$, an application of the divergence theorem shows that

$$
A=\iiint_{R}\left[u_{\infty}-u_{0}+w_{\infty}-w_{0}\right] d V
$$

as one would expect from the conservation principle. If $u_{\infty}=w_{\infty}=0$, $\psi_{\infty}$ is determined by the initial values of $u$ and $w$ and the boundary values of $u$, so that the total quantity of diffusing matter which flows over any part $S_{0}$ of the boundary $\partial R$ is independent of the function $K(u)$. In particular, if a hollow cylinder is saturated under a boundary concentration $C_{0}$ and then removed from the saturating environment, the proportion of its contents which flow through the internal cylindrical boundary is independent of $K(u)$ and the function $f(u, w)$ describing the interaction between the phases $u$ and $w$.

As an example of this method, suppose

$$
\frac{\partial}{\partial x}\left[K(u) \frac{\partial u}{\partial x}\right]=\frac{\partial u}{\partial t}, \quad 0<x<a,
$$

and $u$ satisfies the boundary conditions $u=0$ at $t=0$ on $(0, a)$, while 
$u=C_{0}$ at $x=a$ and $u=0$ at $x=0$ for all $t>0$. The time integral of the flux through unit area of the face at $x=0$ is asymptotic to $F(\infty) t-A$. Since

$$
\frac{\partial}{\partial x}\left[K\left(u_{\infty}\right) \frac{\partial u_{\infty}}{\partial x}\right]=0
$$

we see that

$$
\int_{0}^{u_{\infty}} K(\theta) d \theta=\frac{b}{a} x \text { where } \quad b=\int_{0}^{c_{0}} K(\theta) d \theta,
$$

and hence

$$
F(\infty)=\left[K\left(u_{\infty}\right) \frac{\partial u_{\infty}}{\partial x}\right]_{0}=\frac{b}{a}=\int_{0}^{c_{0}} \frac{K(\theta)}{a} d \theta .
$$

Again,

$$
\frac{\partial^{2} \psi_{\infty}}{\partial x^{2}}=-u_{\infty}, \text { in }(0, a),
$$

while $\psi_{\infty}=0$ at $x=0$ and $x=a$ and therefore

$$
\begin{aligned}
\psi_{\infty} & =-\int_{0}^{x}(x-z) u_{\infty}(z) d z+\frac{x}{a} \int_{0}^{a}(a-z) u_{\infty}(z) d z \\
A & =\left[\frac{\partial \psi_{\infty}}{\partial x}\right]_{0}=\frac{1}{a} \int_{0}^{a}(a-z) u_{\infty}(z) d z=\frac{1}{2 a} \int_{0}^{c_{0}}(a-z)^{2} d u_{\infty} \\
& =\frac{a}{2} \int_{0}^{C_{0}}\left[1-\left(\int_{0}^{u_{\infty}} K(\theta) d \theta / \int_{0}^{c_{0}} K(\theta) d \theta\right)\right]^{2} d u_{\infty} .
\end{aligned}
$$

Evidently $C_{0} a / 2>A>0$.

Let us finally note that the method depends on the fact that $u$ satisfies a conservation law of the form $\nabla^{2} \phi=\partial q / \partial t$ where $\phi$ is a known function of $u$, while $q$ is given initially and at $t=\infty$ and $u$ has known values on $\partial R$.

\section{REFERENCES}

1. H. Daynes, Process of diffusion through a rubber membrane, Proc. Roy. Soc. 97A (1920), 286.

2. R. M. Barrer, Diffusion in and through solids, Chapter 1, Cambridge Univ. Press, New York, 1941.

Applied Mathematics laboratory, Department of Scientific and Industrial Research, Wellington, New Zealand 